\title{
Sterole in Blättern und Chloroplasten
}

\author{
Waldemar Eichenberger und Wilhelm Menke \\ Botanisches Institut der Universität zu Köln \\ (Z. Naturforschg. 21 b, 859—867 [1966] ; eingegangen am 3. März 1966)
}

\begin{abstract}
The nature and quantity of sterols in leaves and chloroplasts of Spinacia oleracea, Antirrhinum majus, and Allium porrum were determined. From dried leaves $0.05-0.18 \%$ free and esterified sterols were isolated, and $0.04-0.09 \%$ from chloroplasts. Although approximately half the lipids of leaves is localized in chloroplasts we found no more than a quarter of leaf sterols in the chloroplasts. The mixture of sterols contains a major sterol and minor sterols in these species. In spinach $\alpha$-spinasterol is the major sterol and in Antirrhinum and Allium $\beta$-sitosterol. Moreover, we established $\Delta^{7}$-stigmastenol and cholesterol in spinach. Besides for free sterols, leaves contain sterol esters and glycosides. Palmitic acid is the binding partner in the sterol esters of spinach, and glucose and mannose are the binding partners in sterol glycosides. Chloroplasts contain the same sterols as leaves do. Only a trace of sterol glycosides could be detected in chloroplast preparations.
\end{abstract}

Im folgenden soll untersucht werden, ob sich die in den Chloroplasten vorkommenden Sterole von den Sterolen der übrigen Zelle unterscheiden. Dabei war zu berücksichtigen, daß sich in der Pflanze neben freien Sterolen auch deren Fettsäureester und Glykoside befinden. $\mathrm{Zu}$ prüfen war außerdem, welcher Anteil der Blattsterole in den Chloroplasten lokalisiert ist. Als Ergebnis sei vorweggenommen, daß wir kein für die Chloroplasten organspezifisches Sterol fanden. Da die Arbeit aus äußeren Gründen unterbrochen werden mußte, haben wir uns ent- schlossen, die bisher vorliegenden Ergebnisse zu veröffentlichen.

\section{Sterolgehalt von Blättern und Chloroplasten}

Im Unverseifbaren der Lipidextrakte aus Blättern und Chloroplastenpräparaten wurden die Sterole mit Digitonin gefällt und nach Spaltung der Digitonide mit Pyridin gravimetrisch bestimmt. Die Ausbeuten für die einzelnen Versuche sind in Tab. 1 zusammengestellt. Die drei untersuchten Arten weisen

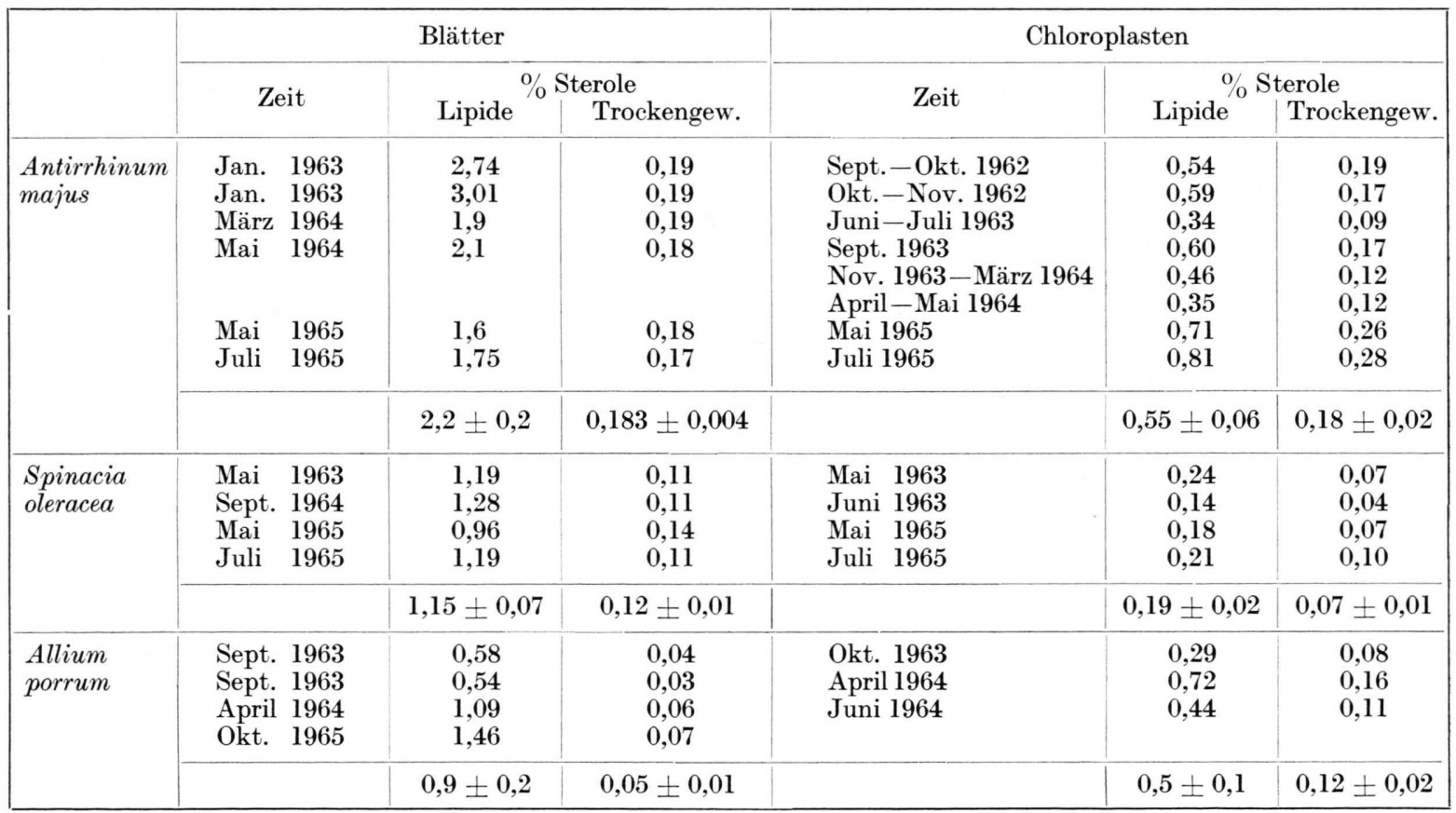

Tab. 1. Sterolgehalt von Blättern und Chloroplasten. 
Unterschiede im Sterolgehalt der Blätter und Chloroplasten auf. Es besteht keine Proportionalität zwischen dem Sterolgehalt der Blätter und dem Sterolgehalt der Chloroplasten. Auch zwischen Lipidgehalt und Sterolgehalt kann keine Proportionalität festgestellt werden. Für die Sterolbestimmung wurden Chloroplasten verwendet, die in wäßrigen Medien isoliert worden waren. Da die Präparate mit dest. Wasser gewaschen wurden, bestanden sie fast ausschließlich aus dem Lamellarsystem der Chloroplasten, welches nach unveröffentlichten Untersuchungen von NickeL ${ }^{1}$ nur etwa $50 \%$ des ChloroplastenTrockengewichtes ausmacht. Die für Chloroplasten angegebenen Zahlen beziehen sich also auf das Lamellarsystem der Chloroplasten. Der wirkliche Sterolgehalt verringert sich also etwa um die Hälfte und kann daher zu 0,04-0,09\% des Trockengewichtes der Chloroplasten angegeben werden. Somit ist der Sterolgehalt der Chloroplasten noch niedriger als früher gefunden wurde ${ }^{2}$. Trotz erheblicher Schwankungen der ermittelten Werte ist eine eindeutige Abhängigkeit der Sterolgehalte von der Jahreszeit und vom Alter nicht festzustellen. Schon aus dem Vergleich der Sterolgehalte in Blättern und Chloroplasten ist zu entnehmen, daß der Hauptteil der Blattsterole nicht in den Chloroplasten lokalisiert ist. Eine quantitative Abschätzung des prozentualen Anteils der Blattsterole, der in den Chloroplasten vorkommt $(\% S)$, ist über den Chlorophyllgehalt der Blätter und Chloroplasten nach der Beziehung

$$
\frac{C_{\mathrm{Bl}} \cdot S_{\mathrm{Ch}} \cdot 100}{C_{\mathrm{Ch}} \cdot S_{\mathrm{Bl}}}=\% S
$$

möglich. In dieser Formel bedeuten $C_{\mathrm{B} 1}$ und $C_{\mathrm{Ch}}$ die Chlorophyllgehalte der Blätter und Chloroplasten und $S_{\mathrm{B} 1}$ und $S_{\mathrm{Ch}}$ die entsprechenden Sterolgehalte. Die Rechnung ergab, daß bei Antirrhinum $18-24 \%$ und bei Spinacia 5-10\% der Sterole in den Chloroplasten lokalisiert sind. Dieser Anteil erscheint niedrig, wenn man berücksichtigt, daß die Chloroplasten etwa die Hälfte der Blattlipide enthalten. Wenn man bedenkt, daß die Lipide der Erythrozyten $20-30 \%$ Sterole $^{3}$ und die Lipide der Nervenzellen 17\% Sterole ${ }^{4}$ enthalten, die in der Erythrozytenmembran bzw. der Markscheide noch angereichert

1 H. NickeL, unveröffentlicht.

2 W. Menke u. E. Jасов, Hoppe-Seiler's Z. physiol. Chem. 272, 227 [1942]. sind, kann ein hoher Sterolgehalt für die Stabilität von lamellar gebauten Lipoproteinsystemen nicht erforderlich sein.

Obschon der größte Teil der Sterole außerhalb der Chloroplasten lokalisiert ist, wurden in den Cuticula-Wachsen von Antirrhinum-Blättern keine Sterole gefunden.

\section{Qualitative Zusammensetzung der Sterolgemische}

Da die Chloroplasten nur sehr wenig Sterole enthalten und Chloroplastenpräparate nur beschränkt zugänglich sind, haben wir zunächst die Blattsterole untersucht. Durch Gaschromatographie ließ sich zeigen, daß die Blattsterole der drei untersuchten Pflanzenarten aus einem Hauptsterol und Nebensterolen bestehen (Abb. 1). Die Sterole von Spinacia oleracea wurden schon mehrfach untersucht. Heyl und Mitarbb. ${ }^{5}$ fanden als Hauptkomponente ein damals

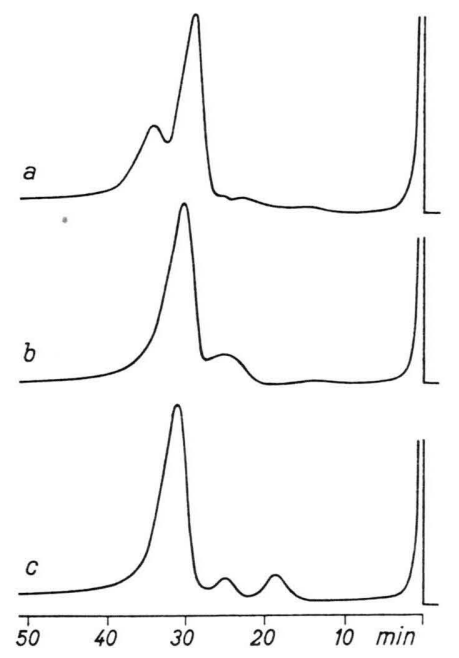

Abb. 1. Gaschromatogramme der Sterole aus Blättern von Spinacia oleracea, Antirrhinum majus und Allium porrum. $\mathrm{SE}-302 \%, 225^{\circ} \mathrm{C}, 120 \mathrm{ml}$ pro Minute. a) Spinacia oleracea, b) Antirrhinum majus, c) Allium porrum.

noch unbekanntes Sterol, das sie als $\alpha$-Spinasterol bezeichneten. Dieses wurde von einer geringen Menge eines ebenfalls neuen Sterols begleitet, das $\beta$-Spinasterol genannt wurde ${ }^{6}$. Das $\alpha$-Spinasterol (I) wurde von Fernholz und Ruigh ${ }^{7}$ und Barton und Cox ${ }^{8}$ als $4^{7,22}$-Stigmastadienol erkannt (Abb. 2). Das

3 W. S. Spector, Handbook of Biological Data, Philadelphia 1956.

${ }^{4}$ R. P. Соок, Cholesterol, Academic Press, New York 1958.

5 F. W. Heyl, E. C. Wise u. J. H. Speer, J. biol. Chemistry 82, 111 [1929]. 


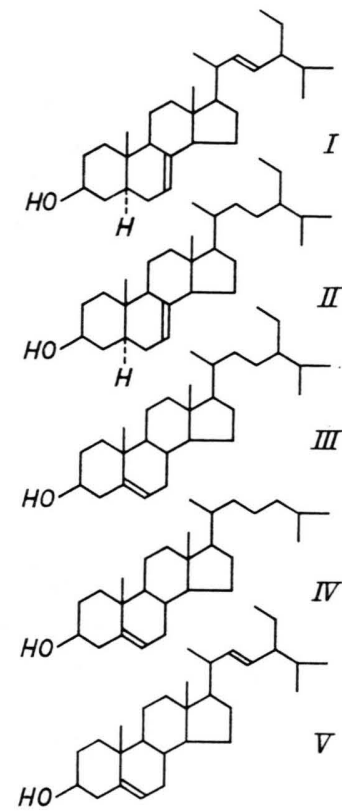

Abh. 2. I $\alpha$-Spinasterol, II $\Delta^{7}$-Stigmastenol, III $\beta$-Sitosterol, IV Cholesterol, V Stigmasterol.

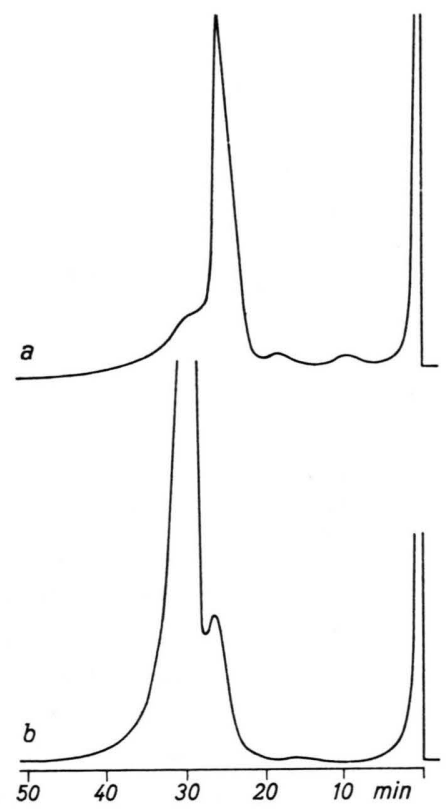

Abb. 3. Gaschromatogramme der angereicherten Sterole aus Spinatblättern. SE-30 $2 \%, 225^{\circ} \mathrm{C}, 120 \mathrm{ml}$ pro Minute. a) a-Spinasterol, b) $\Delta^{7}$-Stigmastenol.

${ }^{6}$ F. W. Heyl u. D. Larsen, J. Amer. pharmac. Assoc., sci. Edit. 22, 510 [1933].

7 E. Fernholz u. W. L. Ruigh, J. Amer. chem. Soc. 62, 2341 [1940].

8 D. H. Barton u. J. D. Cox, J. chem. Soc. [London] 1949, 214.
$\beta$-Spinasterol erwies sich als Gemisch, dessen Hauptkomponente $\Delta^{7}$-Stigmastenol (II) ist ${ }^{9}$. Diese beiden Sterole wurden von uns durch Umkristallisieren und Säulenchromatographie an Aluminiumoxid soweit angereichert, daß sie gaschromatographisch nahezu einheitlich waren (Abb. 3) und identifiziert werden konnten. Abb. 4 zeigt, daß die IR-Spektren eines authentischen Präparates* und unseres Hauptsterols

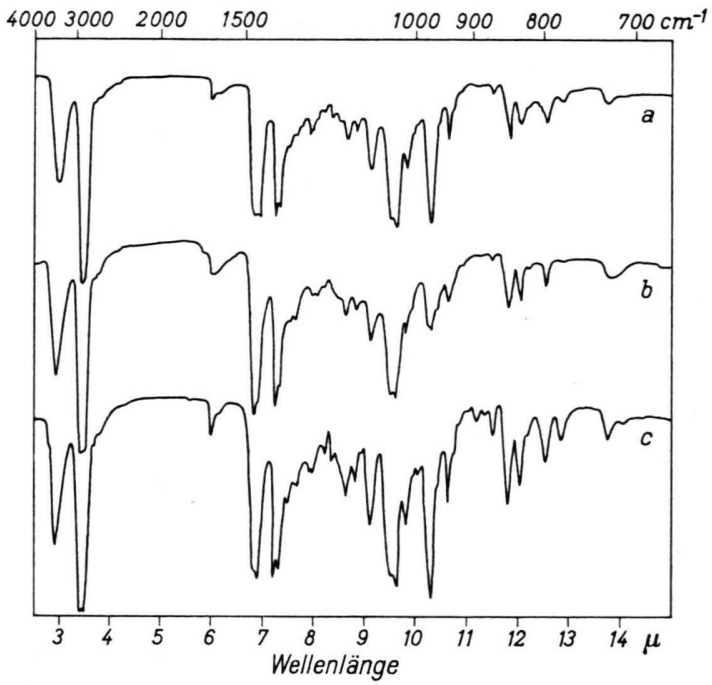

Abb. 4. IR-Spektren der Sterole aus Spinatblättern (in $\mathrm{KBr}$ ). a) $\alpha$-Spinasterol, b) $\Delta^{7}$-Stigmastenol, c) $\alpha$-Spinasterol (authentisch).

aus Spinatblättern übereinstimmen. Beide aus diesen Blättern isolierten Sterole lieferten dasselbe Hydrierungsprodukt.

In der Mutterlauge eines Kristallisationsansatzes trat bei der Gaschromatographie eine Komponente hervor, die dieselbe Retentionszeit besaß wie Cholesterol (IV). Zur näheren Charakterisierung wurde das in der Mutterlauge enthaltene Gemisch bromiert und auf Dünnschichtplatten chromatographiert (Kieselgel G, Benzol-Äthylacetat). Dabei entstand schon bei bloßem Erwärmen der Platte auf $100{ }^{\circ} \mathrm{C}$ bei $R_{f}=$ 0,6 ein blauer Fleck. Derselbe Fleck wurde mit bromiertem Cholesterol, aber auch mit den Bromierungsprodukten von $\beta$-Sitosterol (III) und Stigmasterol (V) erhalten. Dagegen gaben $\alpha$-Spinasterol und $\Delta^{7}$-Stigmastenol diesen Fleck nicht. Daraus kann

9 K. TAkeda u. Y. Matsui, Chem. pharmac. Bull. [Tokyo] 6, 437 [1958].

* Herrn Prof. E. Graf vom Pharmazeutisch-chemischen Institut der Universität Tübingen danken wir für die freundliche Überlassung einer Probe von $a$-Spinasterol. 
man schließen, daß diese Reaktion für $\Delta^{5}$-Sterole charakteristisch ist und daß sich mit ihr $\Delta^{5}$-Sterole von $\Delta^{7}$-Sterolen unterscheiden lassen. Da bei Dünnschichtchromatographie der Bromierungsprodukte von $\alpha$-Spinasterol die Zone um $R_{f}=0,6$ keine Substanzen enthielt, wurde das Bromierungsprodukt aus der oben genannten Mutterlauge eluiert und debromiert. Dabei wird die ursprüngliche Doppelbindung wieder eingeführt. Wie aus Abb. 5 hervorgeht, haben

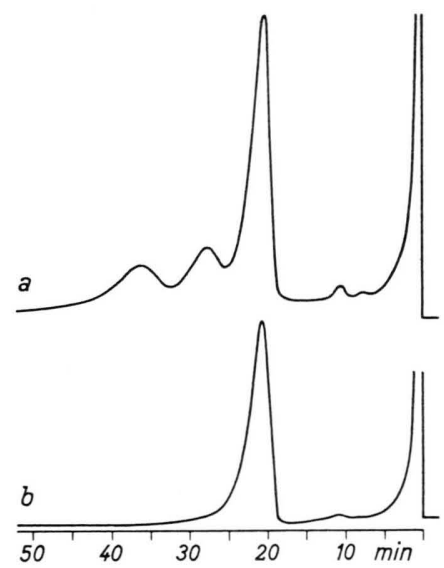

Abb. 5. Gaschromatogramm des Nebensterols aus Spinatblättern. SE-30 $2 \%, 225{ }^{\circ} \mathrm{C}, 120 \mathrm{ml}$ pro Minute. a) Nebensterol (bromiert und debromiert), b) Cholesterol (Sigma).

dieses Produkt und Cholesterin die gleiche Retentionszeit. Da das Produkt ferner im IR-Spektrum dieselben Banden zeigt wie Cholesterol, handelt es sich dabei offenbar um dieses Sterol. Die übrigen Nebensterole konnten noch nicht identifiziert werden. Die Anwesenheit von Cholesterol verdient deshalb Beachtung, weil dieses bis vor wenigen Jahren als ausschließlich tierisches Produkt angesehen wurde. 1958 entdeckte Tsuda Cholesterol in Algen ${ }^{10}$, und neulich wurde es auch in höheren Pflanzen festgestellt ${ }^{11,12}$. Da nach heutiger Auffassung ${ }^{13}$ die Biosynthese der Phytosterole über eine C-27 Zwischenstufe führt, liegt die Vermutung nahe, daß das Cholesterol der Blätter eine solche Zwischenstufe darstellt.

Das Sterolgemisch aus Blättern von Antirrhinum majus unterscheidet sich vom Sterolgemisch aus Blättern von Spinacia durch seine spezifische Drehung. Da sich die Phytosterole nach Angaben von BERGMANN ${ }^{14}$ gemäß ihrer optischen Drehung in fünf

10 K. Tsuda, S. Agaki, Y. Kishida, R. Haiatsu u. K. Sakai, Chem. pharmac. Bull. [Tokyo] 6, 724 [1958].

11 D. F. Johnson, R. P. Bennett u. E. Heftmann, Science [Washington] 140, 198 [1963].
Klassen einteilen lassen, gehören die AntirrhinumSterole mit einer spez. Drehung von etwa $-30^{\circ} \mathrm{zu}$ den $\Delta^{5}$-Sterolen. Nach dem Gaschromatogramm (Abb. 1) liegt auch hier ein Hauptsterol und wenigstens ein Nebensterol vor. Der Anteil der Hauptkomponente beträgt ungefähr 90 Prozent. Das IRSpektrum stimmt weitgehend mit dem des $\beta$-Sitosterols überein. Aus dem Vergleich der optischen Drehung, der Gaschromatogramme und der IRSpektren geht hervor, daß es sich bei dem Hauptsterol aus Blättern von Antirrhinum und Allium porrum ebenfalls um $\beta$-Sitosterol handelt (Abb. 6).

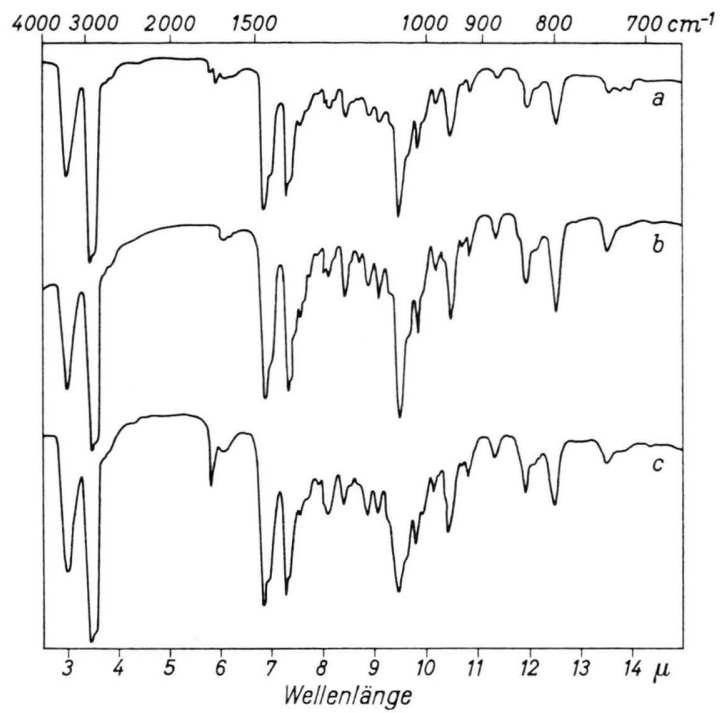

Abb. 6. IR-Spektren der Hauptsterole aus Blättern von Antirrhinum majus und Allium porrum (in $\mathrm{KBr}$ ). a) Allium porrum, b) Antirrhinum majus, c) $\beta$-Sitosterol (Sigma).

Außer freien Sterolen kommen in den Blättern der untersuchten Pflanzen noch gebundene Sterole vor, die zwar eine positive Reaktion nach L i e b e r m a n n-B u r c h a r d geben, aber nicht mit Digitonin fällbar sind (Tab.2). In Blättern von Spinacia und Antirrhinum konnten neben Sterolestern auch Sterylglykoside nachgewiesen werden. Ihre Isolierung gelang durch Säulenchromatographie und Kristallisation. Die betreffende Esterfraktion aus Spinatblättern wurde durch IR-Spektren und durch gaschromatographische Untersuchung der Verseifungsprodukte als Sterolester charakterisiert (Abb. 7). Das IR-Spektrum zeigt neben den typischen Banden

12 J. W. Rowe, Phytochemistry 4, 1 [1965].

13 E. Lederer, Experientia [Basel] 20, 473 [1964].

14 W. Bergmann, Ann. Rev. Plant Physiol. 4, 383 [1953]. 


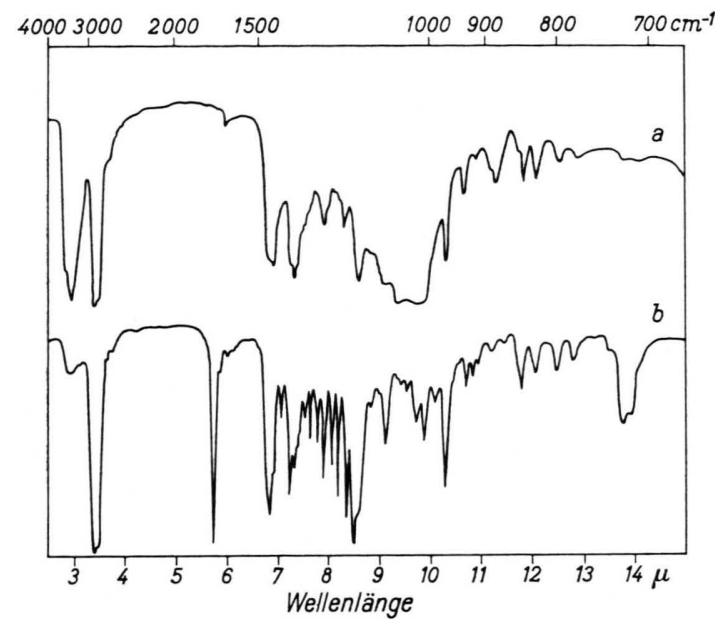

Abb. 7. IR-Spektren gebundener Sterole aus Spinatblättern (in $\mathrm{KBr}$ ). a) Sterylglykosid, b) Sterolester.

des $\alpha$-Spinasterols Esterbanden bei 1740 und $1180 \mathrm{~cm}^{-1}$. Die OH-Bande des freien Sterols bei $3400 \mathrm{~cm}^{-1}$ ist kaum vorhanden. Nach Verseifung wurde $\alpha$-Spinasterol erhalten, das möglicherweise von einer geringen Menge $\Delta^{7}$-Stigmastenol begleitet wird. Gaschromatographie der Methylester der Fettsäuren ergab als Hauptkomponente Palmitinsäure $(88 \%)$. Demnach besteht die Sterolesterfraktion vorwiegend aus $\alpha$-Spinasterylpalmitat. Eine Beteiligung anderer Fettsäuren an der Veresterung der Sterole ist möglich.

\begin{tabular}{|c|c|c|c|c|}
\hline & Datum & $\begin{array}{c}\text { freie } \\
\text { Sterole } \\
{[\%]}\end{array}$ & $\begin{array}{c}\text { Sterole } \\
\text { aus } \\
\text { Estern } \\
{[\%]}\end{array}$ & $\begin{array}{c}\text { Sterole } \\
\text { aus Gly- } \\
\text { kosiden } \\
{[\%]}\end{array}$ \\
\hline Antirrhinum & & & & \\
Blätter & 5.5 .65 & 68 & 7 & 25 \\
Blätter & $\mathbf{7 . 7 . 6 5}$ & 54 & 21 & 25 \\
Chloroplasten & $\mathbf{5 . 5 . 6 5}$ & 78 & 22 & $>0$ \\
Chloroplasten & $\mathbf{7 . 7 . 6 5}$ & 71 & 29 & $>0$ \\
Spinacia & & 57 & 9 & 34 \\
Blätter & 5.5 .65 & 57 & 6 & 25 \\
Blätter & 7.7 .65 & 69 & 41 & $>0$ \\
Chloroplasten & $\mathbf{5 . 5 . 6 5}$ & 59 & 27 & $>0$ \\
Chloroplasten & 7.7 .65 & 73 & 27 \\
\hline
\end{tabular}

Tab. 2. Freie, veresterte und glykosidisch gebundene Sterole in Blättern und Chloroplasten.

Die Sterylglykoside können nach Anreicherung durch Chromatographie wegen ihrer geringen Löslichkeit in den üblichen organischen Lösungsmitteln leicht kristallisiert werden ${ }^{*}$. Wie Abb. 7 zeigt, er-

** Das Sterylglykosid wurde von Herrn A. Röpling zum ersten Mal aus Spinatblättern isoliert und als solches erkannt. gibt das Sterylglykosid aus Spinatblättern die typischen IR-Banden des $\alpha$-Spinasterols bei 968, 842 und $826 \mathrm{~cm}^{-1}$. Bei saurer Hydrolyse wurden a-Spinasterol, $\Delta^{7}$-Stigmastenol und als Zuckerkomponenten Glucose und Mannose im Verhältnis $4: 1$ erhalten (Abb. 10). Da die molaren Verhältnisse der beiden Sterole einerseits und

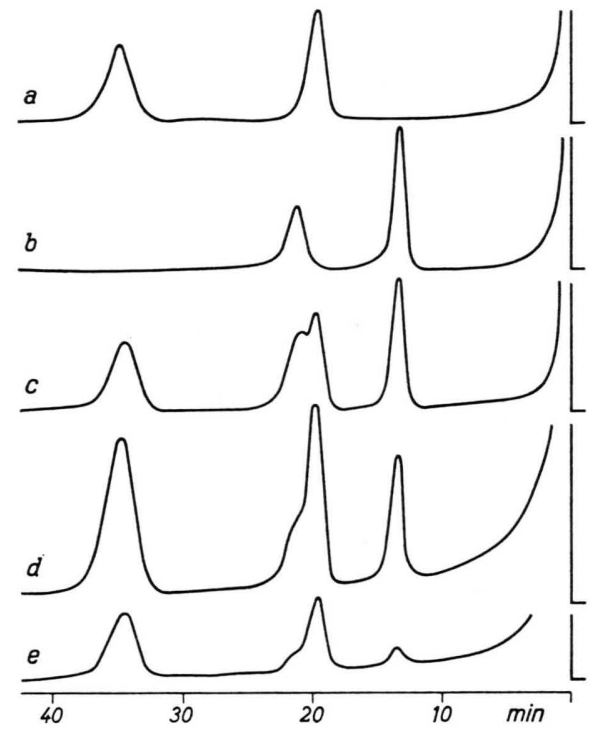

Abb. 10. Gaschromatogramme der Zucker aus Sterylglykosiden (als Trimethylsilyläther). SE-30 $2 \%, 146^{\circ} \mathrm{C}, 75 \mathrm{ml}$ pro Minute. a) Glucose, b) Mannose, c) Glucose+Mannose, d) Spinat-Blätter, e) Antirrhinum-Blätter.

der beiden Zucker andererseits nicht übereinstimmen, wie das beim Vorliegen von nur zwei Glykosiden der Fall sein müßte, kann man damit rechnen, $\mathrm{da} ß$ vier verschiedene Glykoside existieren. Eine entsprechende Untersuchung der Sterylglykoside aus Antirrhinum-Blättern ergab als Aglykon $\beta$-Sitosterol und als Zucker ebenfalls Glucose und Mannose. Die Zucker stehen hier im Verhältnis 6:1. Im weiteren spricht die IR-Absorption für eine $\beta$-glykosidische Verknüpfung von Sterol und Zucker. Bei $882 \mathrm{~cm}^{-1}$ tritt nämlich eine Bande auf, die dieser Bindungsform zugeschrieben werden muß ${ }^{15}$. Bei $\alpha$-glykosidischer Bindung müßte das IR-Spektrum bei $843 \mathrm{~cm}^{-1}$ eine Bande zeigen. Im gleichen Gebiet absorbiert zwar auch das Sterol, doch bestehen im Spektrum der Glykoside keine Anzeichen für eine zusätzliche Absorption in dieser Region. 15 S. A. Barker, E. J. Bourne, R. Stephens u. D. H. Whiffen,
J. chem. Soc. [London] 1954, 3468 . 
Wie aus Abb. 8 hervorgeht, sind die gebundenen Sterole wie die freien Sterole zusammengesetzt.

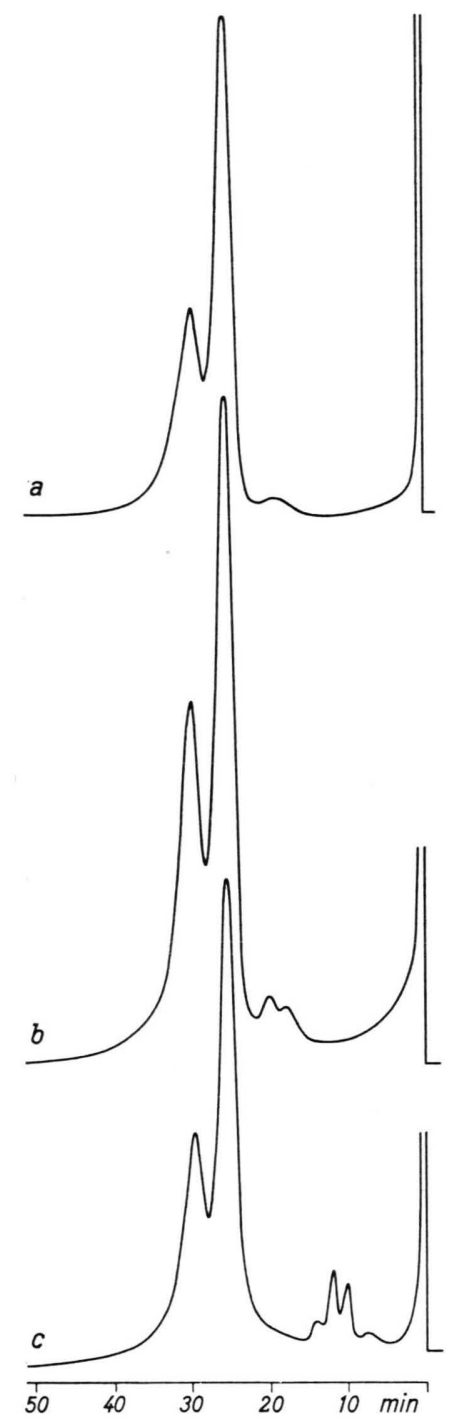

Abb. 8. Gaschromatogramme der freien und gebundenen Sterole aus Spinatblättern. SE-30 $2 \%, 225^{\circ} \mathrm{C}, 120 \mathrm{ml}$ pro Minute. a) Freie Sterole, b) Sterole aus Estern, c) Sterole aus Glykosiden.

In den Chloroplasten konnten die gleichen Sterole wie in den Blättern nachgewiesen werden. Dies ergab sich durch Vergleich der IR-Absorption und der optischen Drehung. Wie Abb. 9 zeigt, sind auch gaschromatographisch keine wesentlichen Unterschiede erkennbar, so daß angenommen werden darf, daß in den Chloroplasten dieselben Sterole etwa im selben Mengenverhältnis vorkommen wie in den Blättern. Außer freien Sterolen fanden wir in den
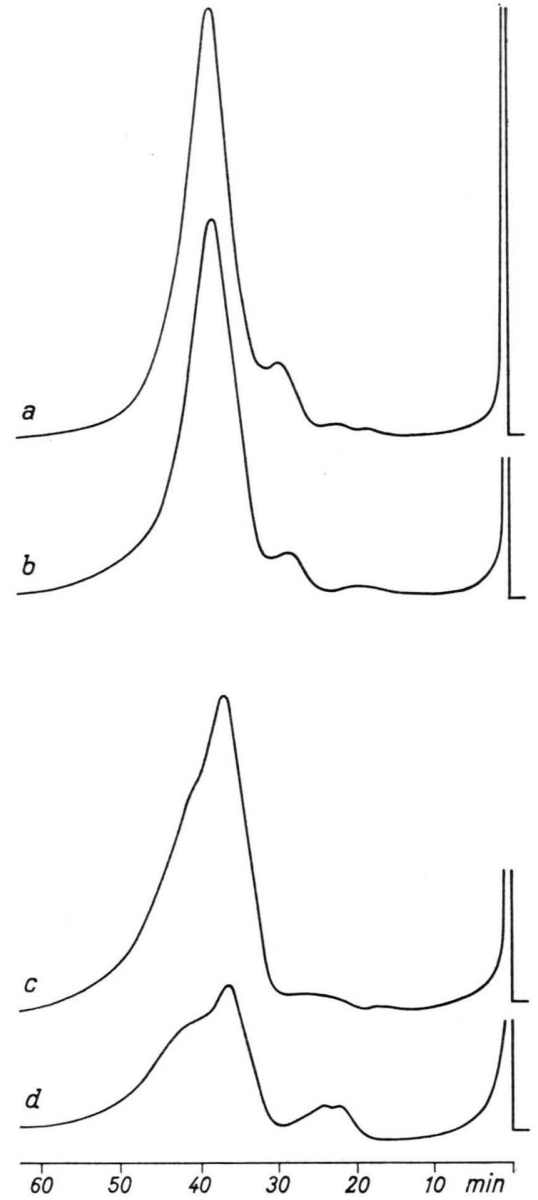

Abb. 9. Gaschromatogramme der freien Sterole aus Blättern und isolierten Chloroplasten von Spinacia oleracea und Antirrhinum majus. SE-30 $2 \%, 225{ }^{\circ} \mathrm{C}, 120 \mathrm{ml}$ pro Minute. a) Antirrhinum-Chloroplasten, b) Antirrhinum-Blätter, c) Spinat-Blätter, d) Spinat-Chloroplasten.

Chloroplastenpräparaten auch veresterte Sterole und in Spuren Glykoside. Der Anteil der veresterten Sterole scheint in den Chloroplasten etwas höher zu sein als in den Blättern (Tab. 2). Da zwischen den Sterolen der Blätter und den Sterolen der Chloroplasten keine sicheren Unterschiede nachgewiesen werden konnten, und da der Gehalt an Sterolen in den Chloroplastenpräparaten sehr niedrig ist, könnte man Zweifel haben, ob die gefundenen Sterole nicht aus Verunreinigungen der Chloroplastenpräparate herrühren. Doch haben Mercer und Mitarbb. ${ }^{16}$ auch in Chloroplastenpräparaten, die in organischen Lösungsmitteln isoliert worden waren,

16 E. I. Mercer, NATO Advanced Study Institute "Biochemistry of Chloroplasts", Aberystwyth 1965, to be published. 
Sterole nachweisen können. Besondere, für Chloroplasten typische Sterole scheinen jedoch nicht zu existieren.

\section{Experimenteller Teil}

Das Pflanzenmaterial stammte größtenteils aus eigener Anzucht. Von Antirrhinum majus wurden Freilandund Gewächshauspflanzen der Sippe 50 verwendet. Für die präparative Darstellung der Sterole aus Spinatblättern wurde eine größere Menge Spinat käuflich erworben.

\section{Isolierung der Chloroplasten}

Die Isolierungsmethode wurde im einzelnen schon früher beschrieben ${ }^{17},{ }^{18}$. Die Chloroplasten wurden aus frisch geernteten und über Nacht im Dunkeln gehaltenen Blättern durch Zermahlen mit 0,4-m. Saccharose und fraktioniertes Zentrifugieren gewonnen.

\section{Isolierung und Trennung der Lipide}

Das gefriergetrocknete Material wurde mit trockenem Methanol und Äther erschöpfend extrahiert. Die in Äther löslichen Lipide wurden im wesentlichen nach der Vorschrift von Lepage ${ }^{19}$ fraktioniert. Als Beispiel sei folgender Ansatz erwähnt: $12 \mathrm{~g}$ Lipide wurden gelöst in $70 \mathrm{ml}$ Heptan (dest.) auf eine Säule von Kieselsäure „Mallinckrodt" $(5,5 \mathrm{~cm} \phi, 11 \mathrm{~cm}$ Höhe) aufgetragen. Durch Eluieren mit $2800 \mathrm{ml}$ Heptan- ̈̈ther 100:1 wurden die Sterolester eluiert (Fraktion I). Danach wurde nacheinander mit $2000 \mathrm{ml}$ Heptan- ̈̈ther $1: 1$ und $700 \mathrm{ml}$ Chloroform eluiert, wobei die freien Sterole ausgewaschen wurden (Fraktion II). AnschlieBend wurde mit Chloroform-Methanol 100:1 weiter eluiert und der Methanolgehalt nach je $500 \mathrm{ml}$ um 1\% erhöht. Die Sterylglykoside wurden mit 2 und 3\% Methanol erhalten (Fraktion III). Die Vollständigkeit der Trennung von Sterolestern und Sterolen wurde jeweils dünnschichtchromatographisch auf Kieselgel G mit Benzol-Äthylacetat 4:1 überprüft. Die Reinheit der Sterylglykoside wurde auf Kieselgel G mit Chloroform-Methanol-Ammoniak (25\%) 75:21:1 kontrolliert. Zur Sichtbarmachung wurde Phosphormolybdänsäure verwendet.

Zur Gewinnung der Cuticula-Wachse wurden die frisch geernteten Pflanzen in Chloroform getaucht.

\section{Isolierung und quantitative Bestimmung der freien Sterole}

Die Lipidfraktion II wurde mit $10 \% \mathrm{KOH}$ in Alkohol $(90 \%) 1$ Stde. am Rückflußkühler verseift. Die unverseifbaren Anteile wurden in Alkohol (90\%) aufgenommen und mit einer Lösung von $1 \%$ Digitonin in

17 W. Menke u. E. Jordan, Z. Naturforschg. 14 b, 234 [1959].

18 W. Kreutz u. W. Menke, Z. Naturforschg. 15 b, 402 [1960].
Alkohol (90\%) gefällt. Die Digitonide wurden mit Pyridin gespalten und die freien Sterole gravimetrisch bestimmt. Bei den meisten quantitativen Bestimmungen wurde wie folgt verfahren: Das aus den ätherlöslichen Lipiden gewonnene Unverseifbare wurde mit Heptan aufgenommen und auf einer Säule von Florisil (Floridin Company) chromatographiert. Für 0,7 bis $1,5 \mathrm{~g}$ Unverseifbares wurde eine 60 bis $65 \mathrm{~cm}$ lange Säule, deren Durchmesser $3,2 \mathrm{~cm}$ betrug, verwendet. Das bei $120^{\circ} \mathrm{C}$ eine Stunde aktivierte Florisil wurde mit 7\% Wasser inaktiviert. Eluiert wurde mit Heptan, dem steigende Mengen Äther zugesetzt wurden. Die Fraktion, die mit Heptan- ̈̈ther $3: 1$ erhalten wurde, enthielt die Sterole, die mit Digitonin gefällt wurden.

\section{Isolierung und Hydrolyse der Sterolester}

$4,73 \mathrm{~g}$ der Fraktion I aus Spinatblättern wurden auf Kieselsäure „Mallinckrodt" $(2 \mathrm{~cm} \phi, 30 \mathrm{~cm}$ Länge $)$ chromatographiert und in folgender Reihenfolge eluiert: $400 \mathrm{ml}$ Heptan, $200 \mathrm{ml}$ Heptan-Äther 100:1, $600 \mathrm{ml}$ Heptan-Äther 100:2, $200 \mathrm{ml}$ Heptan- ̈̈ther $100: 3$. Da sich die Sterolester in den letzten beiden Fraktionen angereichert fanden, wurden deren Rückstände in Heptan auf eine Säule aus Aluminiumoxid „Merck“ (Aktivitätsstufe I) aufgetragen und wie folgt eluiert: $300 \mathrm{ml}$ Heptan, $600 \mathrm{ml}$ Heptan-Benzol $19: 1,500 \mathrm{ml}$ Heptan-Benzol $9: 1,100 \mathrm{ml}$ Heptan-Benzol 1:1. Die Rückstände der letzten drei Fraktionen wurden viermal aus Aceton umkristallisiert. Kristallisat und Mutterlaugen wurden getrennt verseift. Aus $6,8 \mathrm{mg}$ des Kristallisats wurden 4,7 mg Sterole und 2,4 mg Fettsäuren erhalten. Die Fettsäuren wurden mit Diazomethan verestert und auf einer Säule aus Chromosorb W mit $10 \%$ Polyäthylenglykoladipat getrennt. Temperatur $195^{\circ} \mathrm{C}$, Detektorspannung $1000 \mathrm{~V}$, Gasgeschwindigkeit $60 \mathrm{ml}$ Argon pro Minute. Die Sterole wurden an einer Säule aus Gaschrom P mit 2\% SE-30 als stationäre Phase untersucht. Temperatur $225^{\circ} \mathrm{C}$, Detektorspannung $1000-1250 \mathrm{~V}$, Gasgeschwindigkeit $120 \mathrm{ml}$ Argon pro Minute. Im Chromatogramm waren die Gipfel von $\alpha$-Spinasterol und $\Delta^{7}$-Stigmastenol zu erkennen. Zur quantitativen Bestimmung der Sterolester wurde Fraktion I verseift und die Sterole aus dem Unverseifbaren mit Digitonin gefällt.

\section{Trennung von a-Spinasterol und $\Delta^{7}$-Stigmastenol}

Das aus Spinatblättern gewonnene Sterolgemisch wurde 20-mal aus Aceton umkristallisiert. Dabei reicherte sich $\alpha$-Spinasterol im Kristallisat an. Dessen spez. Drehung betrug $[\alpha]_{\mathrm{D}}^{20}=+0,3^{\circ}(\alpha$-Spinasterol $\left.[\alpha]_{\mathrm{D}}=-3,7^{\circ 0}\right)$.

$\mathrm{Zu}$ einer eisgekühlten Lösung von ca. $1 \mathrm{mg}$ Substanz in $2 \mathrm{ml}$ Chloroform wurde $1 \mathrm{ml}$ einer Lösung von 1 Tropfen konz. Schwefelsäure in $1 \mathrm{ml}$ Essigsäureanhy-

19 M. Lepage, J. Lipid Res. 5, 587 [1964].

20 W. Karrer, Konstitution und Vorkommen der organischen Pflanzenstoffe, Birkhäuser-Verlag, Basel 1958. 
drid gegeben (modifizierte Reaktion nach LiebermanNBurchard ${ }^{21}$. Es entstand sofort eine tiefblaue Färbung, die mit $\Delta^{5}$-Sterolen nicht erhalten wurde.

Etwa $1 \mathrm{mg}$ Substanz wurde ferner in 0,4 ml Eisessig gelöst und mit 0,2 $\mathrm{ml}$ einer 2-proz. Lösung von Brom in Chloroform unterschichtet (Reaktion nach ToRTelli$\mathrm{J}_{\mathrm{AFFE}}{ }^{22}$ ). Dabei entstand nach 3 Min. eine grüne Grenzschicht, wie sie auch mit $\alpha$-Spinasterol erhalten wird. Das IR-Spektrum stimmte mit dem von $\alpha$-Spinasterol überein (Abb. 4).

Eine kleine Probe wurde mit Platinoxid in Äthylacetat 4 Stdn. bei Zimmertemperatur hydriert. Aus dem IR-Spektrum des Reaktionsproduktes mußte geschlossen werden, daß die $\Delta^{7}$-Doppelbindung nicht hydriert, sondern bloß verschoben wurde. Da die spez. Drehung des Hydrierungsproduktes mit $[\alpha]_{\mathrm{D}}^{20}=+22,4^{\circ}$ derjenigen von $\Delta^{8(14)}$-Stigmastenol sehr nahe kommt $\left([\alpha]_{\mathrm{D}}^{20}\right.$ $=+24^{\circ} 22$, dürfte es sich beim Hydierungsprodukt um dieses handeln.

Zur Gewinnung des $\Delta^{7}$-Stigmastenols wurde ein Teil der Mutterlaugen der Acetonumkristallisation benzoyliert. $300 \mathrm{mg}$ Sterole wurden in $2 \mathrm{ml}$ trockenem Pyridin gelöst und mit $0,3 \mathrm{ml}$ Benzoylchlorid (dest.) $2 \mathrm{Stdn}$. unter Rückfluß erhitzt und noch $20 \mathrm{Stdn}$. stehengelassen. Nach Verdampfen des überschüssigen Benzoylchlorids im Vakuum wurde der Rückstand aus Aceton umkristallisiert. Die Benzoate wurden in $40 \mathrm{ml} \mathrm{Hep-}$ tan-Benzol 9:1 gelöst und an einer Säule von $30 \mathrm{~g}$ Aluminiumoxid „Merck“ (Aktivitätsstufe I) chromatographiert. Die ersten $150 \mathrm{ml}$ des Eluats enthielten einen gaschromatographisch fast reinen Stoff. Die Drehung betrug $[\alpha]_{\mathrm{D}}^{20}=+10,9^{\circ}\left(\Delta^{7}\right.$-Stigmastenol $\left.[a]_{\mathrm{D}}^{20}=+11^{\circ 22}\right)$. Auch die übrigen Eigenschaften ließen sich mit der Konstitution des $\Delta^{7}$-Stigmastenols zwanglos vereinbaren: Die Hydrierungsprodukte zeigten gleiche optische Drehung und gleiche IR-Spektren wie die von $\alpha$-Spinasterol. Die Reaktionen von $L$ i e. bermann-Burchard und Tortelli-Jaffe verliefen positiv. Das IR-Spektrum unterscheidet sich von dem des $\alpha$-Spinasterols nur durch Abwesenheit der Bande bei $970 \mathrm{~cm}^{-1}$, was für eine gesättigte Seitenkette spricht. Die Retentionszeit ist länger als die von $\alpha$-Spinasterol. Dies entspricht der Beobachtung von TSUDA ${ }^{23}$, daß bei sonst gleichbleibender Konstitution gesättigte Seitenketten längere Retentionszeiten ergeben als ungesättigte.

\section{Isolierung und Nachweis des Cholesterins}

Ein Teil der Mutterlaugen von der Acetonumkristallisation wurde bromiert ${ }^{24} .7,0 \mathrm{mg}$ Sterole wurden in $0,5 \mathrm{ml}$ Äther gelöst und mit $0,15 \mathrm{ml}$ einer 5-proz. Lösung von Brom in Eisessig versetzt. Nach 5 Min. wurde das Reaktionsgemisch auf einer Kieselgel G-Schicht mit Benzol- ̈̈thylacetat $4: 1$ chromatographiert. Beim Erwärmen der Platte auf $100^{\circ}$ entstand bei $R_{f}=0,6$ ein

21 R. P. Соок, A. Kliman u. L. F. Fieser, Arch. Biochem. Biophysics 52, 439 [1954].

22 L. F. Fieser u. M. Fieser, Steroide, Verlag Chemie, Weinheim 1961 . blauer Fleck, der auch mit den Bromierungsprodukten von Cholesterol, $\beta$-Sitosterol und Stigmasterol erhalten wurde. $\alpha$-Spinasterol und $\Delta^{7}$-Stigmastenol dagegen ergaben kein solches Produkt. Zur präparativen Darstellung dieses Bromierungsproduktes aus den Mutterlaugen wurde die entsprechende Zone ohne vorherige Sichtbarmachung von der Platte abgeschabt und mit Äther eluiert. Zur Entbromierung ${ }^{23}$ wurde der Rückstand in $2 \mathrm{ml}$ Eisessig aufgenommen und mit einer Spatelspitze Zinkstaub 20 Min. lang auf dem Wasserbad erhitzt. Nach Filtrieren und Neutralisieren mit Natriumbicarbonat wurde durch Ausschütteln mit Äther ein Reaktionsprodukt gewonnen, das dieselbe Retentionszeit wie Cholesterol zeigte. Auch das IR-Spektrum entsprach dem des Cholesterols.

\section{Isolierung und Hydrolyse der Sterylglykoside}

Die bei der Trennung der Lipide erhaltene Fraktion III wurde erneut auf eine Säule aus Kieselsäure „Mallinckrodt" (1 cm $\varnothing, 10 \mathrm{~cm}$ Höhe $)$ gegeben und mit Chloroform eluiert, dem 6\% Methanol in Stufen von $0,5 \%$ zugesetzt wurden. Die Sterylglykoside fanden sich in den Fraktionen mit 2,5-6\% Methanol und wurden anschließend aus Pyridin umkristallisiert. Das Sterylglykosid aus Spinatblättern zeigte eine spez. Drehung von $[\alpha]_{\mathrm{D}}^{20}=-33^{\circ}$ in Pyridin und ergab auf Kieselgel G-Schichten mit Chloroform-Methanol-Ammoniak $75: 21: 4$ einen einheitlichen Fleck bei $R_{f}=$ 0,44 .

Die Sterylglykoside aus Chloroplasten wurden nach der ersten Anreicherung zur weiteren Reinigung präparativ auf Dünnschichtplatten chromatographiert und anschließend mit 10\% KOH in Alkohol 90\% eine Stde. am Rückflußkühler erhitzt. Das Gemisch wurde mit Schwefelsäure neutralisiert und im Vakuum getrocknet. Der Rückstand ergab auf Dünnschichtplatten in Mischung mit kristallinem Sterylglykosid einen einzelnen Fleck. 13,6 mg Sterylglykosid aus Spinatblättern wurden mit $10 \mathrm{ml} 1-n$. Schwefelsäure 6 Stdn. am Rückfluß erhitzt. Aus dem Reaktionsgemisch wurden durch Ausschütteln mit Äther $9,55 \mathrm{mg}$ freie Sterole gewonnen (theoret. 10,1 mg, berechnet für $\alpha$-Spinasterylglucosid). Die wäßrige Phase wurde mit Bariumhydroxid neutralisiert. Die Zucker wurden auf Schichten aus Cellulosepulver (Macherey-Nagel) mit Butanol-Pyridin-Wasser 6:4:3 chromatographiert und mit Anilinphtalat $(2,5 \%)$ sichtbar gemacht. Dabei entstanden zwei Flecke mit $R_{f}$-Werten von 0,42 und 0,45 , die sich im Mischchromatogramm mit denen von Glucose und Mannose deckten. Die Zucker wurden außerdem als Trimethylsilyläther an einer Säule aus Gaschrom P mit $2 \%$ SE-30 als stationäre Phase gaschromatographisch untersucht. Temperatur $146^{\circ}$, Gasgeschwindigkeit $75 \mathrm{ml}$ Argon pro Minute. Wie Abb. 10 zeigt, stimmen die Retentionszeiten der Produkte genau mit denen überein, die

${ }^{23}$ K. Tsuda, K. Sakai u. N. Inekawa, Chem. pharmac. Bull [Tokyo] 9, 835 [1961].

24 A. Stoll u. E. Jucker, in: K. Paech u. M. V. Tracey, Mod. Meth. d. Pflanzenanalyse, Bd. 3, Springer, Berlin 1955. 
mit Trimethylsilyläthern von Glucose und Mannose erhalten wurden. Die Trimethylsilyläther wurden nach Sweeley et al. ${ }^{25}$ und KaGAN und MaBry ${ }^{26}$ hergestellt. Etwa $2 \mathrm{mg}$ Zucker wurden in $0,5 \mathrm{ml}$ Pyridin gelöst und nacheinander mit $0,1 \mathrm{ml}$ Hexamethyldisilazan und $0,05 \mathrm{ml}$ Trimethylchlorsilan versetzt. Nach Zusatz von $5 \mathrm{ml}$ Chloroform wurde das Gemisch im Vakuum zur Trockne eingedampft und in $1 \mathrm{ml}$ Heptan aufgenommen.

\section{Chlorophyllbestimmung}

Im wesentlichen wurde nach der Vorschrift von Bruinsma 27 verfahren. Die frischen Proben wurden im Mörser mit Seesand zerrieben und mit 80-proz. Aceton extrahiert. Mit einem Zeiss-Spektrophotometer (PM Q II) wurden die Extinktionen bei 663, 652 und $645 \mathrm{~m} \mu$ gemessen. Der Chlorophyllgehalt der Lösung wurde nach den Beziehungen

$$
\begin{aligned}
& \mathrm{mg} \text { Chlorophyll } / l=20,2 \cdot E_{645}+8,02 \cdot E_{663} \\
& \mathrm{mg} \text { Chlorophyll } / l=\frac{1000 \cdot E_{652}}{36}
\end{aligned}
$$

errechnet.

\section{Geräte}

Die gaschromatographischen Untersuchungen wurden auf einem PYE-Argon-Gaschromatographen mit Ionisationsdetektor und Säulen von $4 \mathrm{~mm}$ Durchmesser und $125 \mathrm{~cm}$ Länge durchgeführt. Die IR-Spektren wurden mit einem Perkin-Elmer-Infracord in KaliumbromidPreßlingen aufgenommen. Die optische Drehung wurde mit einem lichtelektrischen Zeiss-Polarimeter gemessen. Wo nichts anderes vermerkt ist, wurde als Lösungsmittel Chloroform verwendet.

Der Stiftung für Stipendien auf dem Gebiet der Chemie, Basel, danken wir für die Gewährung eines Stipendiums an W. Eichenberger. Die Arbeiten wurden mit Unterstützung der $\mathrm{D}$ e u t s chen Forschungsgemeins chaft durchgeführt. Fräulein Schölzel danken wir für die Ausführung der quantitativen Bestimmungen. Fräulein Dr. D. van $\mathbb{W}_{Y K}$ und den Herrn Dr. P. G. Gülz und Dr. A. Radunz danken wir für Ratschläge und Diskussionen.

25 C. C. Sweeley, R. Bentley, M. Makita u. W. W. Wells, J. Amer. chem. Soc. 85, 2497 [1963].

26 J. Kagan u. T. J. Mabry, Analyt. Chem. [New York] 37, 288 [1965].

27 J. Bruisman, Biochim. biophysica Acta [Amsterdam] 52, 576 [1961].

\title{
Elektrophoretische Untersuchungen des „Chlorophyll-Holochroms“ von Nicotiana tabacum L.
}

\author{
Dietrich Düvel \\ Staatsinstitut für Angewandte Botanik, Hamburg \\ (Z. Naturforschg. 21 b, 867-871 [1966]; eingegangen am 23. Mai 1966)
}

\begin{abstract}
The mode of acting in free electrophoresis was studied with a chlorophyll and protein containing extract, prepared by using digitonine. This extract shows an anodic shifting direction. Within the $p_{\mathrm{H}}$-range of about $4,1-8,4$ this complex behaves as an uniform homogeneous substance. At $p_{\mathrm{H}} 9,4$ the main part of the digitonine, that was necessary to dissolve the chlorophyll-proteincomplex, splits off being slower than the green chlorophyll containing fraction, the so called "Chlorophyll-Holochrome". This could be a possibility to isolate this "Chlorophyll-Holochrome" in an aqueous solution, to investigate the structure proteids or even the enzymes of the very first steps in photosynthesis being adjacent to chlorophyll.
\end{abstract}

Die Frage nach den mit dem Chlorophyll gekoppelten Proteinverbindungen ist nicht nur für die Aufklärung der Struktur der chlorophyllhaltigen, submikroskopischen Bauelemente von Bedeutung, sondern sie greift ebenso sehr in die Deutung der ersten Schritte der Photosynthese ein. In Anlehnung an eigene frühere Arbeiten und in Fortführung der Untersuchungen des "Chlorophyll-Holochroms“ von Nicotiana tabacum ${ }^{1}$ sollte zur weiteren Klärung des Verhaltens dieses Pigmentkomplexes auch die Elektrophorese zur Untersuchung herangezogen werden.

1 D. DüveL, Beitr. Biol. Pflanzen 39, 83 [1963].
Die chemischen Untersuchungen sowie auch die Sedimentationsversuche hatten gezeigt, daß es sich bei dem mit Digitonin herausgelösten Pigmentkomplex um Teilchen handeln mußte, die sowohl Rohchlorophyll als auch Protein enthielten. Spektralphotometrische Untersuchungen und vor allen Dingen auch Fluoreszenzuntersuchungen an diesem Komplex hatten deutlich gezeigt, daß sich der mit Digitonin herausgelöste Pigmentkomplex ähnlich wie Rohchlorophyll verhält, das noch von seinen natürlichen Nachbarsubstanzen umgeben war. Bei einem $p_{\mathrm{H}}$-Wert von 8,4 , der dem Wert unter natürlichen Bedingungen wohl recht nahe kommt, konnten die 